\title{
34. DATA REPORT: COMPOSITIONS OF FLUIDS AND AUTHIGENIC PHASES IN SEDIMENTS OF THE NANKAI TROUGH ACCRETIONARY COMPLEX ${ }^{1}$
}

\author{
Miriam Kastner ${ }^{2}$ and Henry Elderfield ${ }^{3}$
}

\begin{abstract}
Pore fluid and sediment chemical and isotopic data were obtained at ODP Site 808, western Nankai trough, Japan. The isotopic data reported here (Tables 1, 2,3,4) were carried out in our shore-based laboratories to complement the shipboard inorganic geochemical data published in Shipboard Scientific Party (1991). The data include: hydrogen, oxygen, and strontium isotopic compositions of the pore fluids, carbon and oxygen isotopic compositions of biogenic and authigenic calcites, and preliminary mineralogical compositions of the authigenic precipitates in veins and in tubular-cylindrical structures, observed in the sediments between 870 and $1080 \mathrm{mbsf}$. These data do not support active pervasive fluid flow at the décollement zone nor at the frontal thrust. They do, however, support pervasive active lateral fluid flow above and particularly below the décollement.
\end{abstract}

\section{INTRODUCTION}

Sediments entering subduction zones typically have porosities of approximately $50 \% \pm 10 \%$, while the porosities of subaerially exposed accretionary complex sediments are less than 5\%-10\%. Large scale fluid expulsion accompanies this major porosity reduction. Internal processes, such as thermal dehydration and decomposition of hydrous minerals, provide additional fluids to the system. While moving through the sediments, the fluids interact with them and transport heat and solutes. Exchange reactions occur between the sediments and the fluids and modify the chemical and isotopic compositions of the fluids and diagenetic solids. Unravelling the origins of these complex, diagenetically altered fluids requires extensive chemical and isotopic analyses of both the pore fluids and of the sediments, especially of the diagenetic precipitates.

\section{MATERIALS AND METHODS}

All pore fluids analyzed were obtained by routine shipboard squeezing of whole-round sediment samples, almost immediately after retrieval. The sediments were squeezed in both titanium and stainlesssteel squeezers, at ambient temperature and at pressures of 2000 $3000 \mathrm{psi}\left(140-210 \mathrm{~kg} / \mathrm{cm}^{2}\right)$. Occasionally, for short periods of time, the most indurated samples were squeezed at $3500 \mathrm{psi}\left(245 \mathrm{~kg} / \mathrm{cm}^{2}\right)$. The Ti squeezers were provided by D. Froelich from Lamont-Doherty Geological observatory. No systematic differences were observed in the chemistry of the fluids obtained by the two different squeezers. Samples were analyzed on board ship for a range of constituents and were supplemented by shore-based analysis summarized in Tables 1 through 4. Oxygen, hydrogen, carbon, and strontium isotopic compositions were determined by mass spectrometry. Stable isotopes in calcites were determined using the method of McCrea (1950). The accuracy was $0.04 \%$ and the precision $0.07 \%$ for carbon and $0.080 \%$ for oxygen. Isotopic compositions of water samples were determined using the method of Bottinga and Craig (1969). Precisions were $0.80 \%$ for $\mathrm{D} / \mathrm{H}$ and $0.10 \%$ for oxygen. $\mathrm{Sr}$ isotopes were determined as previously described, for example by Elderfield et al. (1982). Precisions

\footnotetext{
'Hill, I.A., Taira, A., Firth, J.V., et al., 1993. Proc. ODP, Sci. Results, 131: College Station, TX (Ocean Drilling Program).

${ }^{2}$ Scripps Institution of Oceanography, University of California, San Diego, La Jolla, CA 92093-0212, U.S.A.

${ }^{3}$ Department of Earth Sciences, University of Cambridge, Cambridge CB2 3EQ, United Kingdom.
}

are shown in Tables 1 and 2. Measured ${ }^{87} \mathrm{Sr}{ }^{86} \mathrm{Sr}$ ratios were normalized to ${ }^{87} \mathrm{Sr} /{ }^{86} \mathrm{Sr}=0.1194$, but have not been normalized to a particular value for a standard; values of 0.71027 for NBS 987 and 0.70916 for modern seawater have been obtained.

The mineralogy of the authigenic minerals described in Kastner et al. (this volume) was determined by routine X-ray diffraction (XRD) methods and by scanning electron microscopy (SEM).

\section{Pore Fluid Sr Isotopic Composition of Dried-up Solid Samples}

When sediment porosities are low, $\leq 30 \%$, no pore water is recovered by squeezing the sediment, even from $20-30 \mathrm{~cm}$ long whole round samples. We therefore were unsuccessful in recovering a pore water sample from Core $808 \mathrm{C}-69 \mathrm{R}$, the décollement core. In order to test the feasibility of leaching such "dry" sediments with ultra-pure $\mathrm{H}_{2} \mathrm{O}$ to extract $\mathrm{Sr}$ from pore waters without any $\mathrm{Sr}$ isotopic fractionation and without sampling exchangable $\mathrm{Sr}$ from solids, we conducted the following experiments:

The pore fluids and the leached $\mathrm{Sr}$ of the following dried sediment samples: $808 \mathrm{C}-94 \mathrm{R}-1,0-35 \mathrm{~cm}$, and $808 \mathrm{C}-99 \mathrm{R}-1,0-30 \mathrm{~cm}$, were analyzed for their ${ }^{87} \mathrm{Sr} /{ }^{86} \mathrm{Sr}$ isotopic compositions. These two samples were chosen for the test because of the very large differences between the Sr isotopic compositions of the squeezed on-board pore fluids and of the bulk sediments (Table 1). Based on lengthy testings in our laboratory, these samples were "leached" with ultra-pure $\mathrm{H}_{2} \mathrm{O}$ at room temperature for 3-5 min. The results for $\mathrm{Sr}$ isotopes are given below. "Leaching" dry sediments for longer times or for short times but at moderately elevated temperatures, of $30^{\circ}-60^{\circ} \mathrm{C}$, is not recommended. Some $\mathrm{Sr}$ is being extracted from the solids in the latter pretreatments of sediment samples.

\begin{tabular}{|c|c|c|c|}
\hline & \multicolumn{3}{|c|}{${ }^{87} \mathrm{Sr} /{ }^{86} \mathrm{Sr}$} \\
\hline Sample & $\begin{array}{c}\text { Squeezed } \\
\text { pore water }\end{array}$ & $\begin{array}{l}\text { Leach with } \\
\text { ultra-pure } \mathrm{H}_{2} \mathrm{O}\end{array}$ & Bulk sediment \\
\hline $\begin{array}{l}808 \mathrm{C}-94 \mathrm{R}-1 \\
0-35 \mathrm{~cm}\end{array}$ & $0.708563 \pm 17$ & $0.708625 \pm 20$ & $0715178 \pm 17$ \\
\hline $\begin{array}{l}808 \mathrm{C}-99 \mathrm{R}-1, \\
0-30 \mathrm{~cm}\end{array}$ & $0.707891 \pm 16$ & $0.707961 \pm 19$ & $0.175663 \pm 18$ \\
\hline
\end{tabular}

The effects of either longer "leaching" times even at room temperature, and leaching at $35^{\circ} \mathrm{C}$ are summarized below for Sample $808 \mathrm{C}$ $68 \mathrm{R}-1,5-10 \mathrm{~cm}$ : 
${ }^{87} \mathrm{Sr} /{ }^{86} \mathrm{Sr}$

\begin{tabular}{ll}
\hline Squeezed pore water & ${ }^{87} \mathrm{Sr} /{ }^{86} \mathrm{Sr}$ \\
$\begin{array}{l}\text { "Leached" pore water, room } \\
\text { temperature, 1 hour }\end{array}$ & $0.707622 \pm 20$ \\
"Leached" pore water, $35^{\circ} \mathrm{C}$, & $0.707693 \pm 18$ \\
15 minutes & \\
\hline
\end{tabular}

On the basis of these plus additional tests, we proceeded to analyze the décollement Sample, Core $69 \mathrm{R}-1$, for its pore water $87 \mathrm{Sr} / 86 \mathrm{Sr}$ ratio. The result is given in Tables 1 and 2. This value was obtained by "leaching" two separate subsamples of the composite sediment sample (954-963 mbsf) in ultra-pure $\mathrm{H}_{2} \mathrm{O}$ at room temperature for $3 \mathrm{~min}$. The data from the two leaches are practically identical:

$$
\begin{aligned}
& \text { Analysis } 1: 0.707764 \pm 18 \\
& \text { Analysis } 2: 0.707760 \pm 20
\end{aligned}
$$

The above tests and $\mathrm{Sr}$ isotopic results clearly indicate that even "dry" sediment samples can be reliably analyzed for their pore water Sr isotopic composition.

Table 1. Chemical and $\mathrm{Sr}$ isotopic compositions of pore fluids from the Nankai trough, Site 808.

\begin{tabular}{lccccc}
\hline $\begin{array}{c}\text { Core, section, } \\
\text { interval }(\mathrm{cm})\end{array}$ & $\begin{array}{c}\text { Depth } \\
(\mathrm{mbsf})\end{array}$ & $\begin{array}{c}\mathrm{Cl} \\
(\mathrm{mM})\end{array}$ & $\begin{array}{c}\mathrm{Sr} \\
(\mu \mathrm{M})\end{array}$ & ${ }^{87} \mathrm{Sr}{ }^{86} \mathrm{Sr}$ & $\sigma$ \\
\hline 131-808C- & & & & & \\
3H-5, 140-150 & 23 & 572 & 60 & 0.708711 & 22 \\
5X-1, 88-103 & 151 & 576 & 82 & 0.708592 & 20 \\
19X-1, 135-150 & 284 & 580 & 103 & 0.708367 & 29 \\
11R-2, 50-63 & 397 & 581 & 107 & 0.708348 & 18 \\
27R-5, 123-150 & 557 & 588 & 135 & 0.707723 & 21 \\
28R-2, 124-150 & 562 & 592 & 129 & 0.707797 & 22 \\
53R-4, 120-150 & 805 & 512 & 116 & 0.707099 & 23 \\
55R-2, 120-150 & 822 & 514 & 202 & 0.707069 & 16 \\
60R-4, 116-150 & 873 & 475 & 262 & 0.707121 & 16 \\
61R-2, 115-150 & 879 & 474 & 258 & 0.707164 & 16 \\
64R-1, 115-150 & 907 & 481 & 282 & 0.707281 & 20 \\
66R-2, 115-150 & 928 & 468 & 284 & 0.707499 & 22 \\
68R-1, 5-10 & 944 & 476 & 298 & 0.707622 & 20 \\
39R-1, composite sample & $954-963$ & - & - & 0.707764 & 18 \\
70R-4, 0-35 & 968 & 476 & 302 & 0.707798 & 21 \\
71R-2, 120-150 & 976 & 471 & 277 & 0.707812 & 18 \\
77R-4, 0-35 & 1035 & 465 & 302 & 0.708084 & 21 \\
79R-2, 0-35 & 1050 & 453 & 271 & 0.708183 & 18 \\
84R-1, 120-150 & 1093 & 466 & 283 & 0.708370 & 24 \\
86R-2, 115-150 & 1109 & 447 & 299 & 0.708408 & 16 \\
b4R-1, 0-35 & 1185 & 466 & 372 & 0.708563 & 17 \\
99R-1,0-30 & 1234 & 512 & 500 & 0.707891 & 16 \\
\hline
\end{tabular}

${ }^{\text {a }}$ Wash with ultrapure water of carefully cleaned composite sample.

${ }^{b}$ Solid residue 0.715178 (17)

${ }^{\mathrm{c}}$ Solid residue $0.715663(18)$

\section{REFERENCES*}

Bottinga, Y., and Craig, H., 1969. Oxygen isotope fractionation between $\mathrm{CO}_{2}$ and water and the isotopic composition of marine atmospheric $\mathrm{CO}_{2}$. Earth Planet. Sci. Lett., 5:285-295.

Craig, H., 1961. Isotopic variation in meteoric waters. Science, 133:1702-1703.

Elderfield, H., Gieskes, J.M., Baker, P.A., Oldfield, R.K., Hawkesworth, C.J., and Miller, R., $1982 .{ }^{87} \mathrm{Sr} /{ }^{86} \mathrm{Sr}$ and ${ }^{18} \mathrm{O} /{ }^{16} \mathrm{O}$ ratios, interstitial water chemistry and diagenesis in deep-sea carbonate sediments of the Ontong Java Plateau. Geochim. Cosmochim. Acta, 46:2259-2268.

McCrea, J.M., 1950. On isotope chemistry of carbonates and paleotemperature scale. J. Chem. Phys., 18:849-857.

Shipboard Scientific Party, 1991. Site 808. In Taira, A., Hill, L., Firth, J.V., et al., Proc. ODP, Init. Repts., 131: College Station, TX (Ocean Drilling Program), 71-269.

* Abbreviations for names of organizations and publication titles in ODP reference lists follow the style given in Chemical Abstracts Service Source Index (published by American Chemical Society).

Date of initial receipt: 6 January 1992

Date of acceptance: 28 September 1992

Ms 131SR-117

Table 2. Sr Isotopic ratios and concentrations of pore fluids from the

\begin{tabular}{|c|c|c|c|c|c|c|c|}
\hline $\begin{array}{l}\text { Core, section, } \\
\text { interval (cm) }\end{array}$ & $\begin{array}{l}\text { Depth } \\
\text { (mbsi) }\end{array}$ & ${ }^{87} \mathrm{Sr}{ }^{20 t} \mathrm{Sr}$ & $\sigma$ & $\begin{array}{c}\mathrm{Sr}^{2} \\
(\mu \mathrm{M})\end{array}$ & $\underset{(\mu \mathrm{M})}{\mathrm{St}^{b}}$ & t/St & $1 / S r^{4}$ \\
\hline \multicolumn{8}{|l|}{ 131-808C. } \\
\hline $3 \mathrm{H}-5,140-150$ & 23 & 0.708711 & 22 & 60 & 60 & 0.0167 & 0.0167 \\
\hline $5 X-1,88-103$ & 151 & 0.708592 & 20 & 82 & ${ }^{\circ}(83)$ & 0.0122 & $(0.0120)$ \\
\hline $19 \times-1,135-150$ & 284 & 0.708367 & 29 & 103 & (103) & 0.0097 & $(0.0097)$ \\
\hline IIR $-2.50-63$ & 397 & 0.708348 & 18 & 107 & 107 & 0.0093 & 0.0093 \\
\hline $27 R-5,123-150$ & 557 & 0.707723 & 21 & 135 & 135 & 0.0074 & 0.0074 \\
\hline $28 \mathrm{R}-2,124-150$ & 562 & 0.707797 & 22 & 129 & (15i) & 0.0078 & $(0.0066)$ \\
\hline $53 \mathrm{R}-4,120-150$ & 805 & 0.707099 & 23 & 116 & (108) & 0.0086 & $(0.0093)$ \\
\hline $55 R-2,120-150$ & 822 & 0.707069 & 16 & 202 & 202 & 0.0050 & 0.0050 \\
\hline $60 R-4,116-150$ & 873 & 0.707121 & 16 & 262 & 262 & 0.0038 & 0.0038 \\
\hline $61 R-2,115-150$ & 879 & 0.707164 & 16 & 258 & 258 & 0.0039 & 0.0039 \\
\hline $64 \mathrm{R}-1.115-150$ & 907 & 0.707281 & 20 & 282 & 282 & 0.0035 & 0.0035 \\
\hline $66 \mathrm{R}-2.115-150$ & 928 & 0.707499 & 22 & 284 & 284 & 0.0035 & 0.0035 \\
\hline $68 R-1.5-10$ & 944 & 0.707622 & 20 & 298 & (325) & 0.0034 & $(0.0031)$ \\
\hline $69 \mathrm{R}-1$, composite sample & $954-963$ & 0.707764 & 18 & - & - & - & - \\
\hline $70 R-4,0-35$ & 968 & 0.707798 & 21 & 302 & 302 & 0.0033 & 0.0033 \\
\hline $7 \mid R-2.120-150$ & 976 & 0.707812 & 18 & 277 & 277 & 0.0036 & 0.0036 \\
\hline $77 \mathrm{R}-4,0-35$ & 1035 & 0.708084 & 21 & 302 & 302 & 0.0033 & 0.0033 \\
\hline $79 \mathrm{R}-2,0-35$ & 1050 & 0.708183 & 18 & 271 & 271 & 0.0037 & 0.0037 \\
\hline $84 \mathrm{R}-1,120-150$ & 1093 & 0.708370 & 24 & 283 & 283 & 0.0035 & 0.0035 \\
\hline $86 \mathrm{R}-2.115-150$ & 1109 & 0.708408 & 16 & 299 & 299 & 0.0033 & 0.0033 \\
\hline $94 \mathrm{R}-1.0-35$ & 1185 & 0.708563 & 17 & 372 & 372 & 0.0027 & 0.0027 \\
\hline $99 \mathrm{R}-1.0-30$ & 1234 & 0.707891 & 16 & 500 & 500 & 0.0020 & 0.0020 \\
\hline
\end{tabular}
Nankai trough, Site 808 .

"Values from atomic absorption measurements.

balues from atomic absorption measurements except for bold values, measured by isotope dilution. 'Calculated from concentration values measured by atomic absorption.

${ }^{4}$ Calculated from concentration values measured by atomic absomption and isotope dilution.

'The concentrations in parentheses were analyzed by isotope dilution, the rest by atomic absorption

Solid residue ${ }^{81} \mathrm{Sr} r^{* / 6} \mathrm{Sr}$ ratio: $0.715178(17)$

SOlid 
Table 3. Oxygen and hydrogen isotopic compositions of pore fluids from the Nankai trough, Site 808.

\begin{tabular}{|c|c|c|c|c|c|}
\hline \multirow{2}{*}{$\begin{array}{l}\begin{array}{l}\text { Core, section, } \\
\text { interval }(\mathrm{cm})\end{array} \\
131-808 \mathrm{C} \text { - }\end{array}$} & \multirow[t]{2}{*}{$\begin{array}{l}\text { Depth } \\
\text { (mbsf) }\end{array}$} & \multicolumn{2}{|c|}{$\begin{array}{c}\delta^{18} \mathrm{O} \\
(\% \circ \text { SMOW })\end{array}$} & \multicolumn{2}{|c|}{$\begin{array}{c}\delta D \\
(\% \circ \text { SMOW })\end{array}$} \\
\hline & & & & & \\
\hline $3 \mathrm{H}-5,140-150$ & 23 & & & -4.4 & \\
\hline $5 X-1,88-103$ & 151 & -2.5 , & ${ }^{a}-2.3$ & -5.7 , & -5.4 \\
\hline $12 \mathrm{R}-1,130-150$ & 406 & -1.7 & & & \\
\hline $28 \mathrm{R}-4,110-150$ & 565 & -2.2 , & ${ }^{a}-2.2$ & & ${ }^{a}-6.7$ \\
\hline $35 R-4,132-150$ & 632 & -3.3 & & -7.2 , & -7.6 \\
\hline $45 \mathrm{R}-4,125-150$ & 728 & -3.0 & & & \\
\hline $53 \mathrm{R}-4,120-150$ & 805 & -2.1 & & -7.5 , & -9.2 \\
\hline $54 \mathrm{R}-2,120-150$ & 812 & & & -8.9 & \\
\hline $55 \mathrm{R}-2,120-150$ & 822 & -1.9 & & & \\
\hline $61 \mathrm{R}-2,115-150$ & 880 & -2.0 & & & \\
\hline $64 \mathrm{R}-1,115-150$ & 907 & & & -8.8 & \\
\hline $65 \mathrm{R}-2,0-34$ & 917 & -1.1 & & & \\
\hline $66 \mathrm{R}-2,115-150$ & 928 & -0.9 & & & \\
\hline $71 R-2,120-150$ & 976 & -0.7 & & & \\
\hline $72 \mathrm{R}-3,0-5$ & 986 & & ${ }^{a}-0.6$ & -10.3 & a -11.3 \\
\hline $75 \mathrm{R}-1,117-145$ & 1013 & -0.7 & & -9.9 & -9.6 \\
\hline $77 \mathrm{R}-4,0-35$ & 1035 & -0.4 & & -11.7 , & -11.6 \\
\hline $79 \mathrm{R}-2,0-35$ & 1050 & & & -10.0 & \\
\hline $80 \mathrm{R}-2,125-150$ & 1061 & & & -10.8 & -10.5 \\
\hline $84 \mathrm{R}-1,120-150$ & 1093 & -0.2 & & -10.4 & -10.6 \\
\hline $85 R-2,120-150$ & 1101 & & & -10.3 & \\
\hline $86 \mathrm{R}-2,115-150$ & 1111 & -0.1 & & -6.8 , & -8.4 \\
\hline $94 \mathrm{R}-1,0-35$ & 1185 & & & -10.4 & -10.2 \\
\hline $96 \mathrm{R}-1,0-36$ & 1204 & & & -7.8 & -7.6 \\
\hline
\end{tabular}

"Data from Dr. S. Epstein's laboratory.

Table 4. Oxygen and carbon isotopic compositions of diagenetic calcites from Nankai trough sediments, Site 808 .

\begin{tabular}{|c|c|c|c|c|c|}
\hline $\begin{array}{l}\text { Core, section, } \\
\text { interval }(\mathrm{cm})\end{array}$ & $\begin{array}{l}\text { Depth } \\
\text { (mbsf) }\end{array}$ & $\begin{array}{c}\delta^{13} \mathrm{C} \\
(\% \circ \mathrm{PDB})\end{array}$ & $\begin{array}{c}\delta^{18} \mathrm{O} \\
(\% \circ \mathrm{PDB})\end{array}$ & $\begin{array}{c}\delta^{18} \mathrm{O} \\
(\% \circ \mathrm{SMOW})\end{array}$ & Comments \\
\hline \multicolumn{6}{|l|}{$131-808 \mathrm{C}$ - } \\
\hline $20 \times-2,31-33$ & 290 & 0.87 & -0.31 & 30.54 & Bulk sample, in thrust zone \\
\hline $64 \mathrm{R}-4,2-4$ & 910 & -11.25 & -13.25 & 17.20 & Precipitate on slickenside surface \\
\hline $70 \mathrm{R}-1,28-30$ & 964 & -2.99 & -3.10 & 27.66 & Bulk sample \\
\hline $80 \mathrm{R}-\mathrm{CC}, 16-19$ & 1066 & -20.81 & -14.51 & 15.91 & Clear large $(\sim 1 \mathrm{~cm}$ thick $)$ calcite vein \\
\hline $80 \mathrm{R}-\mathrm{CC}, 18-19$ & 1066 & -21.93 & -14.13 & 16.29 & Small clear calcite piece \\
\hline
\end{tabular}

